\title{
THE RESEARCH OF PADDY RICE MOISTURE LOSSLESS DETECTION BASED ON L-M BP NEURAL NETWORK
}

\author{
Jun Sun $^{1,2^{*}}$, Hanping Mao ${ }^{1}$, Jinjuan Liu ${ }^{2}$, Bin Zhang ${ }^{3}$
}

${ }^{1}$ Key Laboratory of Modern Agricultural Equipment and Technology, inistry of Education \& Jiangsu Province, Jiangsu University, Zhenjiang, Jiangsu Province, China 212013

${ }^{2}$ School of Electrical and Information Engineering ,Jiangsu university, Zhenjiang ,Jiangsu Province, China 212013

${ }^{3}$ ChangZhou Institute of Technology, Changzhou, Jiangsu Province, China 213002

* Corresponding author, Address: School of Electrical and Information Engineering, Jiangsu University, Zhenjiang,212013,Jiangsu,Province,China,Tel:(0)13775544650,fax:05118878 03 11,Email:sun2000jun@ujs.edu.cn

Abstract: The method of the quantitative analysis on the paddy rice moisture condition is studied, which is based on the spectral reflectivity of the leaf crest layer. Several subsections are carried on the entire spectrum curve by the equidistance, The sensitive characteristic wave-length is selected based on the table of molecular spectrum sensitive wave band, obtains the characteristic spectral reflection index value to take as the characteristic value. The convergence rate of the BP neural network is slow, so the L-M algorithm is introduced to carry on the renewal of the neural network weights. The paddy rice water moisture quantitative analysis forecast model is established by making use of the fast study function of the L-M algorithm neural network. The forecasting results indicate that the highest prediction error of the paddy rice water content is $6.72 \%$ and the average error rate is $4.23 \%$. The prediction effect is better than the traditional BP network arithmetic, and it can be used in the lossless inspection of paddy rice moisture.

Keyword: Paddy rice; Characteristic wave-length; Spectrum reflection index ;L-M BP Network 


\section{INTRODUCTION}

The paddy rice is one of the most important grain crops in the world, and its all cultivated area and the whole output are only inferior to the wheat. Because the $80 \%$ of populations in the Asian and the 1/3 of the populations in the African and Latin America take the paddy rice as the principal food, the paddy rice production is important outstandingly to the developing countries. In our country, the average rice production of every year occupies the first one in the world in the nearly 10 years, and the paddy rice sown area occupies the $22.8 \%$ of the world total sown area. The average per acre output of the paddy rice approximately is 380 kilograms, which is to be among the most ones in main production paddy rice's country. Because the water content is the main component of paddy rice, the loss of the water content is one of the most universal factors which limits paddy rice primitive forces. The water content influences immediately paddy rice's physiological biochemistry process and shape structure, thus it affects the final paddy rice output and the quality, therefore the water content management is one of the most important measures in the crops production. The prompt and accurate monitoring on or diagnosis on paddy rice water content is very important to raising the paddy rice water content management level and the instruction of saving water of agricultural production.

Domestic and foreign countries have researched paddy rice's water content examination. Michio and so on monitors the water content scarcity condition of paddy rice using the $960 \mathrm{~nm}$ place derivative spectrum(Michio S, 1989). Tian Yongchao and so on have studied the relativity of the paddy rice's crown level near-infrared spectral reflectivity and the water condition of plant under the different holard nitrogen condition(Tian, 2005). The reports about the research using the L-M neural network algorithm to the paddy rice's water content condition quantitative analysis have not been seen in domestic and foreign countries at present.

\section{EXPERIMENTAL DESIGH AND EXPERIMENT}

\subsection{Experimental design}

The experiment of growing paddy rice is carried on in big shed in the agricultural engineering research institute of Jiangsu University, and the selection of paddy rice species is new fragrant superior 80 , whose vegetative cycle is 115 days. Paddy rice is planted in the environment of the greenhouse sand bonsai, keeping the line distance of rows as $0.3 \mathrm{~m} \times 0.3 \mathrm{~m}$. The paddy 
rice's nourishment liquid configuration is according to international paddy rice nourishment liquid formulation.

The paddy rice seeds are sow on July 1, 2007. They are divided into 3 groups according to 3 water content level on August 1. The first group guarantees the sufficient water content supply in entire experiment period. The second group keeps the water immersing the sand just in experiment period. The third group keep the sand on the water scarcity condition in the experiment period.

\subsection{Measure instrument and method}

The instrument used in this experiment is the FieldSpec 3 hand-hold portable spectrum instrument which is produced in American ASD Corporation. This instrument's spectrum measuring range is $350-2500 \mathrm{~nm}$. The sampling interval is $1.4 \mathrm{~nm}$ in the $350-1000 \mathrm{~nm}$ spectrum area, and the resolution is $3 \mathrm{~nm}$. In the $1000-2500 \mathrm{~nm}$ spectrum area the sampling interval is $2 \mathrm{~nm}$, the resolution is $10 \mathrm{~nm}$, the angle of view is 15 degrees. In the spectral region it has 512 array element light PDA array detectors, the concave achromatism diffraction grating, the even field image formation, the easy wave length demarcation, and the sensitivity high characteristics.

The measurement experiment is carried on the sunny and non-clouds weather, the measuring time is 11:00-13:00. The contributory data are collected at the same time, such as the measuring environment parameter, the measuring time, gathering location and so on. Pokes head is vertical downward, goes against $0.8 \mathrm{~m}$ to the adult plant crown level, the data are gathered in the identical place each time. The standard operation procedure is to be optimized, to collect dark current, to measure the white tabula, measuring the terrain feature. Each time the data measured the white tabula are taken as the relative reflectivity (standard tabula rasa reflectivity is 1). The system program must be optimized one time each 10 minutes in order to guarantee the data quality.

The measurement is repeated 3 times in every measuring point, and the average data of 3 spectrum data are taken as the characteristic spectral reflectivity data.

In the visible light and short-infrared regions, with the water content rising, the spectral reflectivity drops. The paddy rice's crown level spectral reflectivity is shown as Fig.1. In visible light region, the wavelengths region of $450-470 \mathrm{~nm}, 540-570 \mathrm{~nm}, 700-730 \mathrm{~nm}$ have the obvious reflection peak, and have high correlativity. In the short-infrared region, near $930-970 \mathrm{~nm}$, $1430 \mathrm{~nm}-1460 \mathrm{~nm}$ and $1630 \mathrm{~nm}-1670 \mathrm{~nm}$ spectral reflectivity also has very high relevance with the leaf water content. The average data of the spectral reflectivity in the above six regions are taken as the characteristic value. 


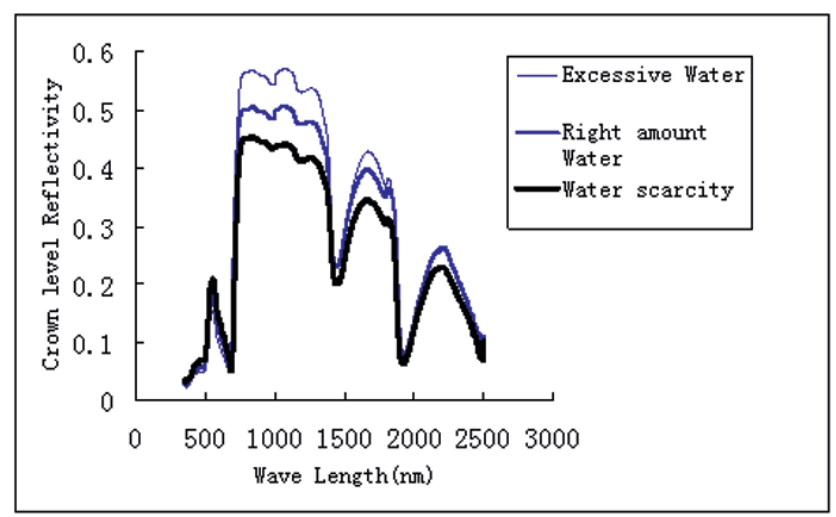

Fig1. The figure of the paddy rice's crown level spectral reflection rate

\subsection{The leaf water contents}

After spectrum measures, the paddy rice leaves are selected randomly and maintained in the keeping freshness bags. Then them are brought back to the laboratory to gain fresh weight and are dried for 1 hour with the constant temperature $100^{\circ} \mathrm{C}$, afterward they are carried on 12 hour being dried with the constant temperature $60{ }^{\circ} \mathrm{C}$, so the leaves' dry weight are measured separately. The calculating formula of leaf water content is shown as formula 1 . The part experiment data of paddy rice leaf water content data are shown as Fig.1.

$$
z=\frac{y-x}{y}
$$

$\mathrm{Z}$ denotes the leaf's water content rate,y denotes the leaf fresh weight, and $\mathrm{x}$ denotes the leaf dry weight.

Table 1. The partial experiment data of paddy rice leaf water content

\begin{tabular}{lllll}
\hline \multirow{2}{*}{ Water volume } & \multicolumn{4}{c}{ Date } \\
\cline { 2 - 5 } & 07.9 .27 & 07.10 .4 & 07.10 .9 & 07.10 .16 \\
\hline Water scarcity & 0.7546 & 0.7292 & 0.7089 & 0.686 \\
Right amount water & 0.7974 & 0.7701 & 0.7533 & 0.7357 \\
Excessive water & 0.7856 & 0.7584 & 0.744 & 0.7159 \\
\hline
\end{tabular}

\section{L-M ALGORITHM BP NETWORK}

The BP neural networks is one kind of multi-layer-forward neural networks which is used most widely. The BP algorithm uses a gradient descent searching method, whose parameter moves along with the opposite direction to error gradient. The error between the practical output and the 
expected output of neural network through computing is computed. Backward each networks weight value is regulated until the error catch the smallest. Its shortcoming lies in its complexity of computation, and its speed is very slow. It possibly falls into the local minimum point. The training time is long, its value stability is bad. Otherwise, the initial weight value, learning rate and momentum coefficient parameters and so on are adjusted difficultly.

The L-M algorithm is one kind of the fast algorithms using standard value optimization techniques. It combines the gradient descent method and Gauss Newton's method, may also take it as the improvement form of a Gauss Newton's method, it has both local convergence of a Gauss Newton's method and the global characteristic of the gradient descent method. Because the L$\mathrm{M}$ algorithm has used the approximate two-rank differential coefficient information, it is much quicker than the gradient method.

The search direction used in the Levenberg -Marquardt's method is the following group of linear equality solution:

$$
\left(J\left(x_{k}\right)^{T} J\left(x_{k}\right)+\lambda_{k} I\right) d_{k}=-J\left(x_{k}\right) F\left(x_{k}\right)
$$

Among them: the scalar $\lambda_{k}$ decides the search direction and amplitude size, when $\lambda_{k}=0$, the search direction is same as the GaussNewton method's; When $\lambda_{k}$ tends to infinity, $d_{k}$ tends to zero vector, thus finds the most quickly descent direction, so long as $\lambda_{k}$ is big enough, it may guarantee $F\left(x_{k}+d_{k}\right)<F\left(x_{k}\right)$, therefore $\lambda_{k}$ can guarantee the function value drop in iteration.

In order to control the size question of $\lambda_{k}$ in each iteration, we linearly predict sum of squares $F_{p}\left(x_{k}\right)$ and cubic interpolated value of the smallest $F_{k}\left(x^{*}\right)$ to estimate relative non-linearity of $F(x)$. The linear prediction sum of squares carries on the computation according to the equation below:

$$
\begin{aligned}
& F_{p}\left(x_{k}\right)=J\left(x_{k-1}\right)^{T} d_{k-1}+f(x) \\
& f_{p}\left(x_{k}\right)=F_{p}\left(x_{k}\right)^{T} F_{p}\left(x_{k}\right)
\end{aligned}
$$

Among them: $f_{k}\left(x^{*}\right)$ and the length of stride parameter $\alpha^{*}$ may through cubic interpolated value $F\left(x_{k}\right)$ and $F\left(x_{k-1}\right), \alpha^{*}$ is the minimum estimated length of stride. If $f_{p}\left(x_{k}\right)$ is bigger than $f_{k}\left(x^{*}\right), \lambda_{k}$ will be reduced to $\frac{\lambda_{k}}{1+\alpha^{*}}$, otherwise ${ }^{\lambda_{k}}$ will be increased to $\lambda_{k}+\frac{f_{k}\left(x^{*}\right)-f_{p}\left(x_{k}\right)}{\alpha^{*}}$. 
With the renewal of $\lambda_{k}$, the solution of formula (1) constitutes a search direction $d_{k}$, then $d_{k}$ increases a unit iteration length of stride to carry on the next search step, so analogizes, this linear search process guarantees $f\left(x_{k+1}\right)<f\left(x_{k}\right)$ in iteration each time. This method has better robustness than the GaussNewton's method(Yuan,2007; Zhang 2004).

\section{EXPERIMENTAL RESULTS}

Because the big shed's air temperature, humidity as well as six spectral interzone's average spectral reflectivity value are taken as the characteristic parameters, therefore neural networks' input layer node number is 8 , the implicit layer node number takes 15 , the output layer node number takes 1.The LM algorithm is applied in the neural networks' weight value renewing, and the network is set up and trained.

The 200 samples are selected to carry on the experiment, among them, 120 samples are taken as the training samples of LM algorithm neural network, and the residual 80 samples are taken as the test samples. Real water content and forecast water content contrast figure based on LM BP network is shown as Fig.2, and the real water content and forecast water content contrast chart based on BP network is shown as Fig.2. From Fig.1, we can see that the points corresponding the real water content and the forecast water content approach a line. It also illuminates that the forecast water content is very near to the real water content. The forecasting results indicate that the highest prediction error of the paddy rice water content is $6.72 \%$ and the average error rate is $4.23 \%$.

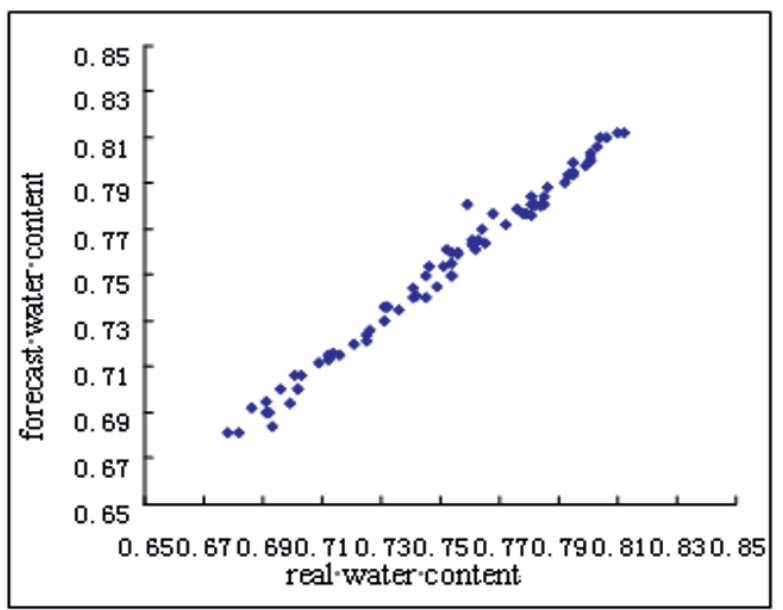

Fig. 2 real water content and forecast water content contrast figure based on LM BP network 


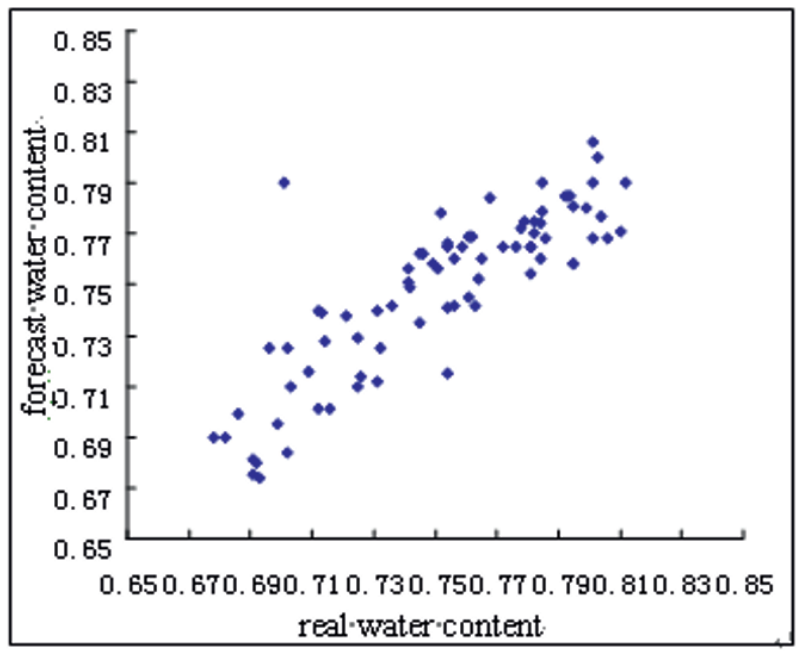

Fig. 3 The real water content and forecast water contentcontrast chart based on BP network

\section{CONCLUSION}

In this paper, the L-M algorithm is imported into BP network to carry on the renewal of the neural network weights. And put the improved BP network into the prediction of the paddy rice water content. The prediction effect is better than the traditional BP network arithmetic. It can provide the reliable water-loss basis for paddy rice planters and it can be used in the lossless inspection of paddy rice moisture. It also has the very significant practical value.

\section{ACKNOWLEDGEMENTS}

Funding for this research was provided by China Postdoctoral Science Foundation under Grant (NO:20070420972); Jiangsu University High-grade Specialty Person Scientific Research Foundation under Grant(NO: 05JDG050); “863"Project(2008AA10Z204); Changzhou Young Scientific and Technological Talent Training Plan (NO:CQ2008009); Jiangsu University College Student Scientific Research Project(NO: 07A087). 


\section{REFERENCES}

Ji Hai-yan, WANG Peng-xin, YAN Tai-lai. Estimations of Chlorophyll and Water Contents in Live Leaf of Winter Wheat with Reflectance Spectroscopy. Spectroscopy and Spectral Analysis, 2007,27(3):514-516.(in Chinese)

Michio S,T suyoshi A. Seasonal visible near-infrared and mid-infrared spectral of rice canopies in relation to LAI and above ground dry phytomass. Remote Sensing of Environment, 1989,27:119-127

Tian Yongchao, CAO Weixing, JIANG Dong, ZHU Yan. Relationship between Canopy Reflectance and Plant Water Content in Rice under different Soil Water and Nitrogen Conditions. 2005, 29(2):318-323(in Chinese)

Yuan Jin-li,GUO Zhi-tao.Stored-grain pests classification based on L-M neural networks. Agricultral Network Information. 2007(6):29-32(in Chinese)

Zhang Bing, Yuan Shouqi, Cheng Li. Model for predicting crop water requirements by using L- M optimization algorithm BP neural network. Transactions of the Chinese Society of Agricultural Engineering. 2004 (6):73-76(in Chinese) 